\title{
THE FEM SIMULATION OF DIESEL INJECTOR NOZZLE DRILLING
}

\author{
Mian Gong ${ }^{1}$, Zihao Ding ${ }^{2}$ \\ ${ }^{1}$ Lecturer, School of Information Technology \& Mechanical and Electrical Integration, Shanghai ZhongQiao College \\ of Vocational Technology, Shanghai, China \\ ${ }^{2}$ Department of Mechanical and Automobile Engineering, Shanghai University of Engineering Science, Shanghai,
} China

\begin{abstract}
With the increasingly strict requirements for diesel engine emissions and the maturity of high-pressure common rail injection technology, the requirements for nozzles in the industry are also increasing. The quality of the nozzle hole has great influence on the performance of the diesel engine. Therefore, how to improve the quality of nozzle hole processing and improve production efficiency has become a problem to be solved. In this paper, based on the theory of high-speed metal micro-cutting, using digital design and computer simulation, the drilling of diesel engine injector nozzle holes is studied. The research contents include the chip formation mechanism and the characteristics of drilling process. The research objectives are as follows: based on the experiments of nozzle hole drilling, the relationship between drilling characteristics and drilling parameters in the process of nozzle hole drilling is studied; the formation process of nozzle hole chip is studied. In drilling simulations, 20CrMo is used as workpiece material and carbide micro-bit is used to process the nozzle holes. Third Wave Advantedge finite element simulation software is used in the simulation.
\end{abstract}

Keywords: - Diesel engine, Drilling, FEM

\section{INTRODUCTION}

The performance, fuel consumption and NOx emissions of diesel engine depend to a large extent on the shape of fuel spray, the size distribution of fuel particles and the temperature, pressure and movement of air in the combustion chamber, which is closely related to the diameter, shape, roughness and discharge coefficient of the nozzle. If there is no mature theoretical basis for nozzle processing and processing technology, the nozzle is not able to achieve good and stable performance. Therefore, it is very important for the processing of nozzle holes. Drilling experiments is usually utilized to test the quality of nozzle processing. Since the cost of drilling experiment is fairly high, simulation is introduced to perform the test.

Finite Element Method (FEM) simulation of machining process requires the knowledge of several different fields, ranging from modern mathematics, mechanics to computer technology and so on. The finite element technology is also a data simulation technology developed with modern science and technology. Finite element technology greatly improves the simulations accuracy of practical cases. It can be used to analyze and evaluate the mechanical performance of various complex mechanical structures, which is valuable reference and analysis. Many software has finite element simulation function, such as ThirdWave, Deform, ANSYS, SolidWorks and so on.

In this paper, the finite element simulation software Third Wave AdvantEdge is used to simulate the drilling of diesel injector nozzle. The mechanism of chip formation, and drilling force variation is summarized. The drilling simulations with different drilling parameters combination are carried out, and the chip formation process, drilling temperature characteristics and drilling force are analyzed accordingly.

AdvantEdge FEM software is a powerful CAE software, which includes the design, establishment, improvement and optimization of processing technology. It enables users to accurately determine the processing parameters and tool configuration, which can reduce cutting force, temperature and deformation effectively. All simulation processes in the software are carried out in off-line state. Using AdvantEdge FEM can effectively reduce the time and cost of online testing. The main advantages of AdvantEdge FEM are:

- $\quad$ Reducing expensive cutting experiments. The software is able to simulate the cutting force, stress field distribution, temperature field distribution, chip shape, chip deformation, residual stress and tool wear in micro and macro aspects.

- The automatic mesh generation only needs to define the mesh control coefficients of cutters and workpieces and the mesh self-adapting redrawing coefficients.

- Optimizing drilling process can optimize spindle speed, feed rate, rake angle and withdrawal speed, prolong tool life and reduce tool wear, improve tool geometry and cutting control, and reduce machining deformation caused by cutting heat, cutting force and residual stress.

- Abundant post-processing functions, curves, cloud pictures and animation show the simulation results. The results of cutting force, temperature, stress, strain rate and machining power can be obtained. It can improve production efficiency and equipment utilization, increase material removal rate and speed up the processing process. 
In practical engineering problems, the difference of the shape and structure of the object leads to the complex force situation of the object, which is difficult to solve in exact function form. In the process of solving a large number of practical engineering problems, researchers have established three numerical methods for solving the force of the object in practical engineering: variation method, difference method and finite element method [1]. In the second half of the twentieth century, the finite element method (FEM) has been developed vigorously and effectively combined with computer high-speed computing technology. It has successfully achieved the required calculation accuracy and has been effectively applied to many practical engineering problems. Today, the finite element analysis method has become the most effective method to solve various large and complex structural problems. Courant proposed the concept of finite element method in 1943 and Turner in 1956 respectively. Clough proposed the name of finite element method in 1960. This method was first applied to plane problems of Aeronautical structures. The essence of finite element analysis is to discretize the solution structure. A complex continuum structure is divided into finite number, finite size and regular shape elements. The nodes between the boundary of each element are connected in a certain way to form a whole discrete structure. The finite element method (FEM) has good solvable characteristics and can be applied to the analysis of various complex engineering structures. It has good generality and can be applied to the solution of different environments and complex structures. It has the characteristics of high accuracy and can meet the requirements of practical engineering by improving the mesh density of structural elements. The finite element method is used to solve structural problems. The calculation process is very complex and huge, which is handled by computer. The actual process of finite element analysis is generally as follows:

- Establish geometric models for cutting tools and workpieces. Before meshing, physical models need to be established. There are two kinds of physical models: one is to import physical models from outside, the other is to generate physical models by setting parameters.

- Structural mesh generation. According to the actual structure of the object, accuracy requirements, actual force conditions and so on, the computer will divide the grid according to certain rules. According to the different strain conditions, the mesh cells are triangular and quadrilateral. The triangular mesh can be well adapted to the boundary conditions and complex shape structure, and the quadrilateral is suitable for the regular shape mesh.

- External environment and condition setting. Conditions setting is the key of finite element analysis. The actual structural stress is calculated according to the actual external conditions.

- $\quad$ Finite element calculation. After dividing the grid and setting the external conditions, the software is used to solve the calculation process.

- Post processing of calculation results. The results of simulation are usually displayed in cloud diagram.

\section{MODELING OF DRILLING SIMULATIONS}

\subsection{Drill Parameters Setting}

The drill bit geometry parameters can be set in the tool setting menu. The bit type used in simulations is standard twist drill. First, select the tool material. Choose common carbide in material menu. The drill bit material used in the actual processing experiment is GU10F superfine carbide. Next, set the geometric parameters of the drill bit. After testing, when the diameter of drill bit is less than $1 \mathrm{~mm}$, the simulation will not be carried out because of the minimum number of units in Advantedge meshing. Therefore, in Fig.1, the diameter of twist drill is set to be $2 \mathrm{~mm}$, the helix angle is 30 degrees, and the apex angle is 130 degrees. Then, the specific parameters of meshing are set in the advanced options menu. The minimum unit size, edge length and meshing gradient are set to be $0.03 \mathrm{~mm}, 0.0002 \mathrm{~mm}$ and 0.4 respectively.

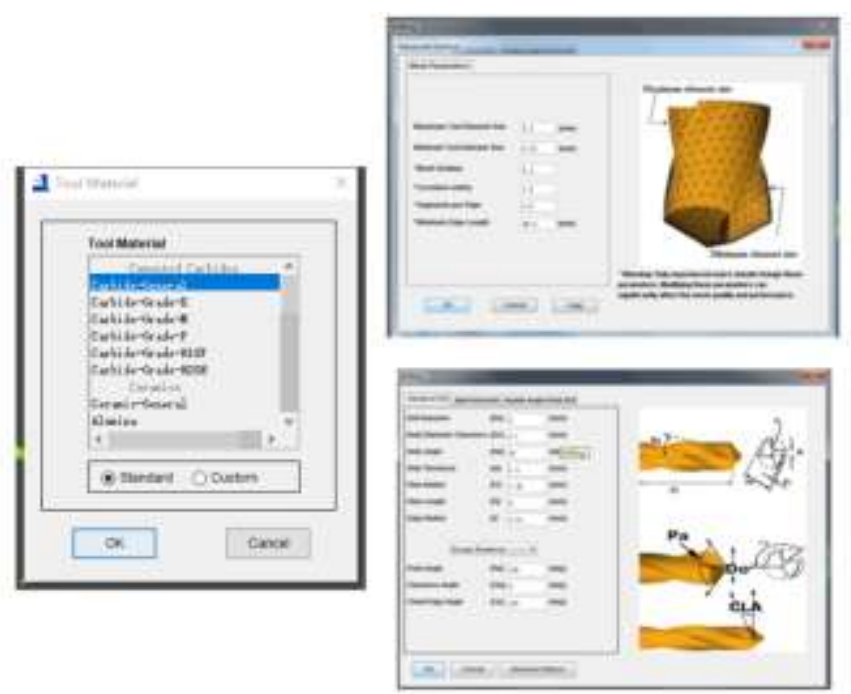

Fig -1: Drill parameters setting

\subsection{Drilling Parameters Setting}

As shown in Fig.2, according to the experimental scheme, drilling parameters including the spindle speed, feed per turn, initial temperature and the angle of bit rotation are set in the menu of drilling process parameters.

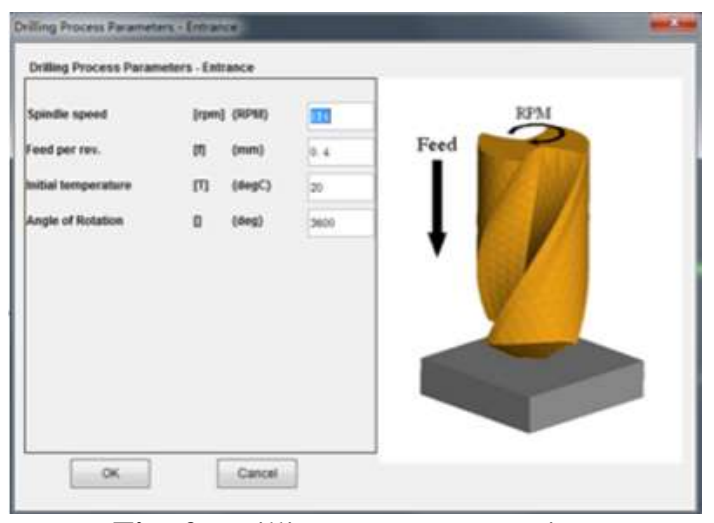

Fig -2: Drilling parameters setting 


\subsection{Workpiece Parameters Setting}

The material of the workpiece is selected in the workpiece menu. Since 20CrMo is not included in the Advantedge stock material library, an alternative is selected as workpiece material. The alloy steel $25 \mathrm{CrMo} 4$ has similar structural and machining properties with $20 \mathrm{CrMo}$, so it is selected as the material of the workpiece as shown in Fig.3. The workpiece dimensions are $12 \mathrm{~mm}$ in length and width, $1 \mathrm{~mm}$ in thickness as shown in Fig.3. Mesh grid options including element size and edge length is set in the advanced options menu.
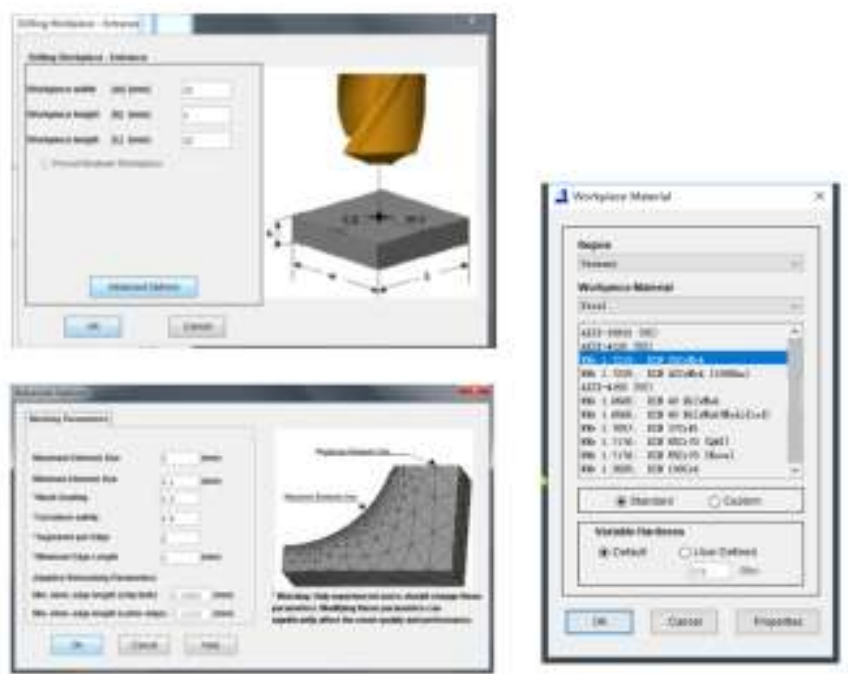

Fig 3: Workpiece parameters setting

\section{DRILLING PROCESS}

\subsection{Cutting Conditions: $10000 \mathrm{r} / \mathrm{min}, 0.2 \mathrm{~mm} / \mathrm{r}$}

After the drill bit cuts into the workpiece, the chip rises along the spiral groove and begins to roll sideways after the formation of the rake face. Therefore, this part of the chip is in spiral shape. Afterwards, under the effect of centrifugal force, the chip ends are thrown outward and the chip ends are left out of the spiral groove. At this time, there are still new chips continuously generated, the length of chips increased. The chip strip the bit, the part of the chips winding the bit is c-shaped. The chip broke and was thrown out. The chip was $\mathrm{C}$ shaped and tail helical. Axial force and torque rise rapidly to the maximum value when the bit touches the workpiece surface. With the increase of drilling depth, the axial force decreases gradually, while the torque keeps the maximum value, and then decreases rapidly after the bit drills out of the workpiece.
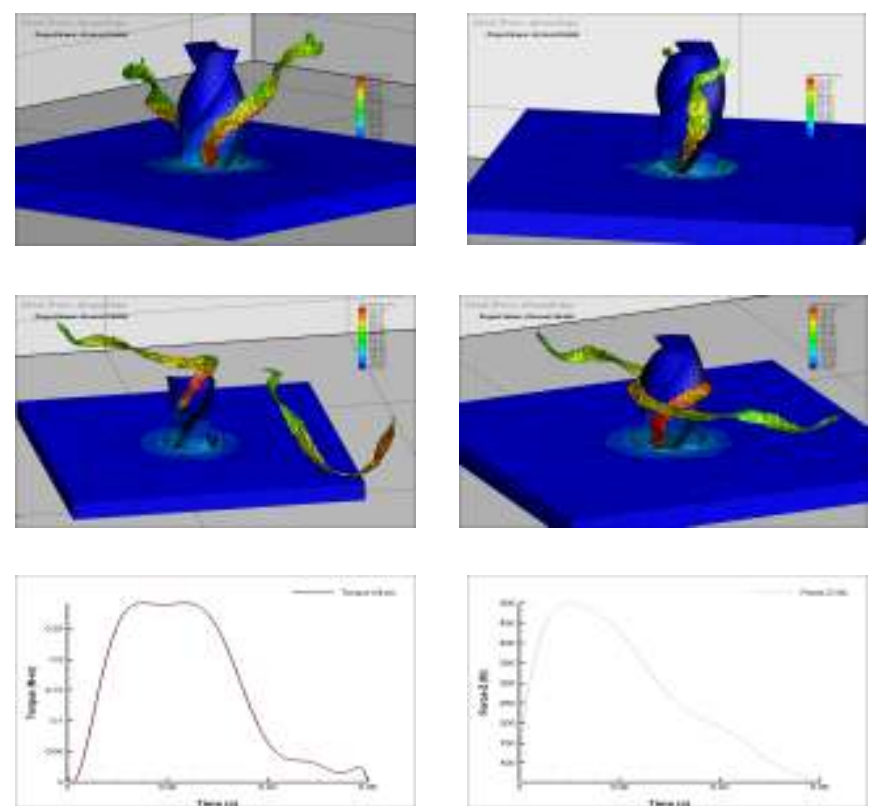

Fig 4: Drilling diagrams (condition 1)

\subsection{Cutting Conditions: 10000r/min, $0.4 \mathrm{~mm} / \mathrm{r}$}

After drilling into the workpiece, there is no obvious chip generation in the spiral groove. After one turn of the cutting edge on one side, the burr edge appears at the entrance of the drill hole. At this time, chips begin to appear in the spiral groove. After a certain distance, the chips break off and fly out, and the chip shape is fan-shaped. The entrance burr edge breaks under the joint extrusion of chips and drills, forming burrs and producing fewer debris-like chips. In the aspect of axial force and torque, both the rising and falling speed are faster, and the drilling force has a smaller peak at the end of the decline. The possible reason is that the chip discharge speed is slower, which leads to slight blockage.
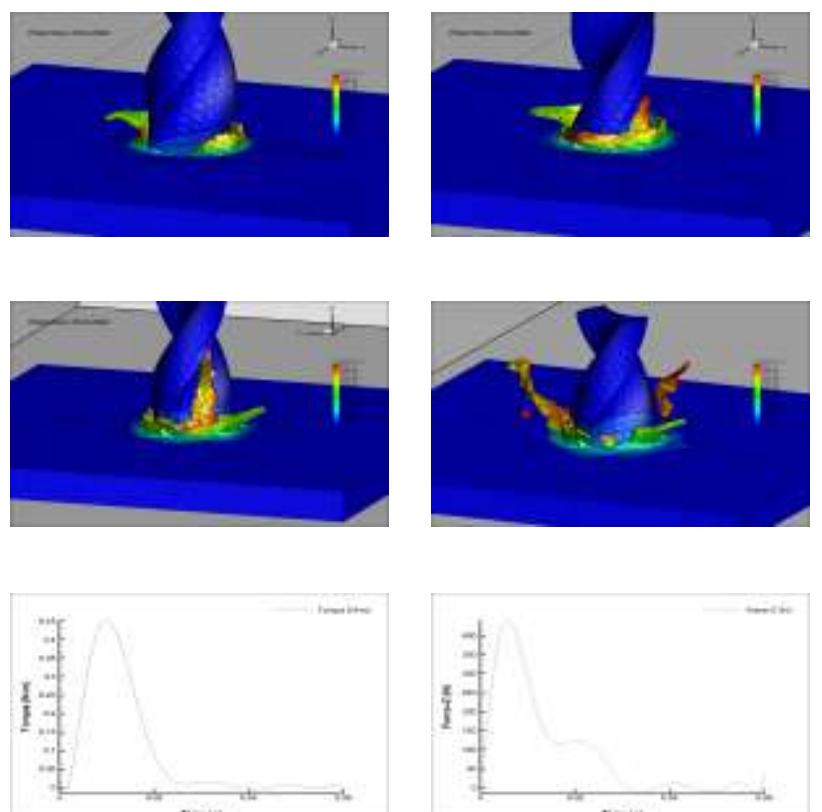

Fig 5: Drilling diagrams (condition 2) 


\subsection{Cutting Conditions: $13000 \mathrm{r} / \mathrm{min}, 0.4 \mathrm{~mm} / \mathrm{r}$}

When the speed is $13000 \mathrm{r} / \mathrm{min}$ and the feed is $0.4 \mathrm{~mm} / \mathrm{r}$, the drilling force is similar to that of $10000 \mathrm{r} / \mathrm{min}$ and $0.4 \mathrm{~mm} / \mathrm{r}$, and the drilling time is shorter. The chip shape is similar to that of $10000 \mathrm{r} / \mathrm{min}$, and the difference is that the exit burr height becomes larger.
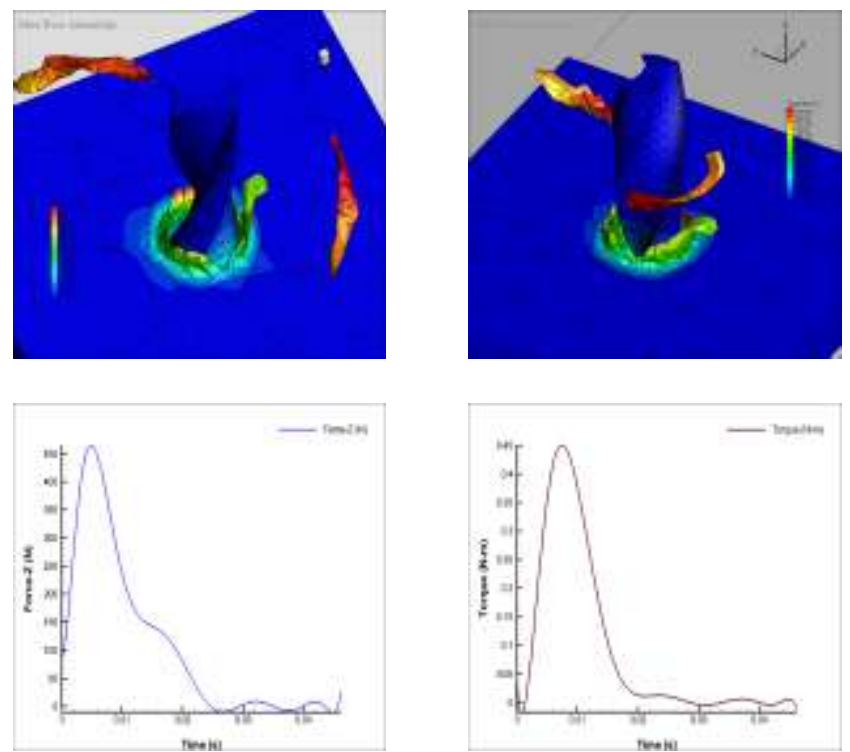

Fig 6: Drilling diagrams (condition 3)

\subsection{Cutting Conditions: 15000r/min, $0.2 \mathrm{~mm} / \mathrm{r}$}

When the speed is $15000 \mathrm{r} / \mathrm{min}$ and the feed is $0.2 \mathrm{~mm} / \mathrm{r}$, the drilling force is similar to $10000 \mathrm{r} / \mathrm{min}$ and $0.2 \mathrm{~mm} / \mathrm{r}$, and the cutting time is shorter. As the rotational speed increases, the rising speed of chips in the spiral groove increases, and the part of the spiral groove contacting the side roll increases, so the shape of chips is closer to the spiral type.
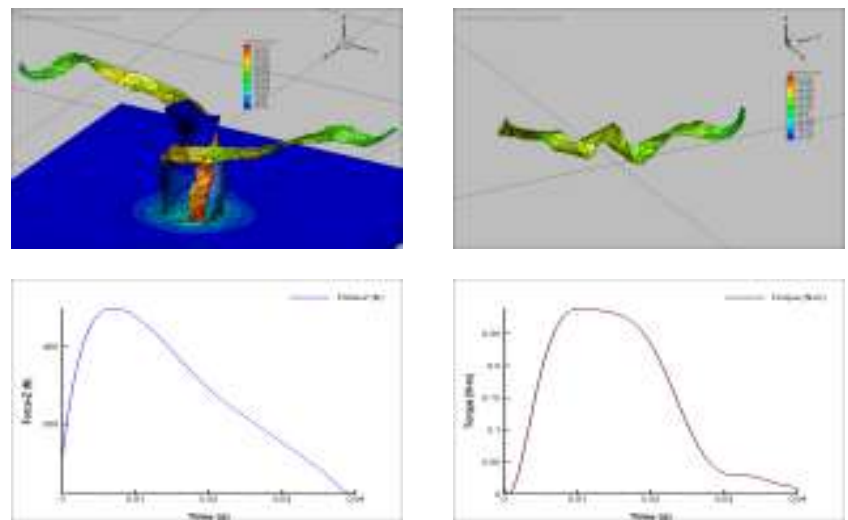

Fig 7: Drilling diagrams (condition 4)

\subsection{Cutting Conditions: 15000r/min, $0.6 \mathrm{~mm} / \mathrm{r}$}

When the rotational speed is $15000 \mathrm{r} / \mathrm{min}$ and the feed rate is $0.6 \mathrm{~mm} / \mathrm{r}$, the drilling force waveform is a single narrow wave. The chip shape is short strip chip, and the exit burr height is larger than that of $0.4 \mathrm{~mm} / \mathrm{r}$ feed. A similar situation occurs when the feed rate is constant and the speed is $13000 \mathrm{r} / \mathrm{min}$.
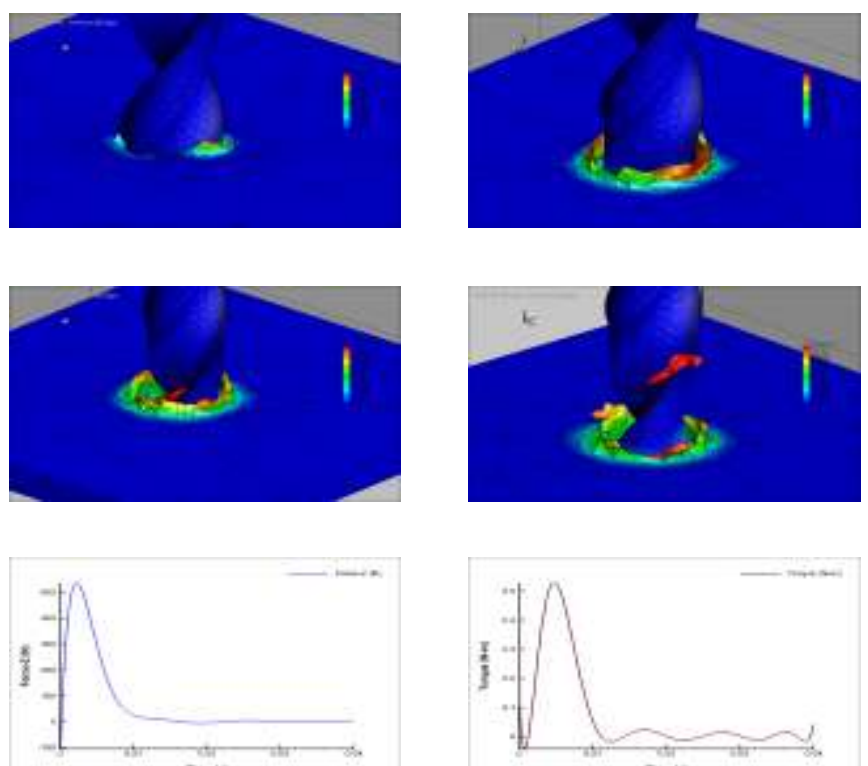

Fig 8: Drilling diagrams (condition 5)

\section{CONCLUSION}

Increasing in feed speed can improve productivity, but when the feed speed is too high, it will increase the workload of cutting edge and chip removal will become difficult. However, low feed speed will not only reduce productivity, but also increase the time of drilling a hole, which will make the temperature in the hole continuously rise, and then increase bit wear. Since the rigidity and strength of the micro drill decrease, it is more necessary to select the feed rate carefully. With the increase of feed speed, the axial drilling force increases gradually. This is because the increase of feed speed is equivalent to the increase of feed per turn while the spindle speed remains unchanged. The chip thickness increases with the increase of feed, and the resistance cutting force of the material increases, so the axial drilling force increases accordingly.

The axial drilling force decreases with the increase of rotational speed. This is because keeping the feed speed unchanged and increasing the speed will greatly reduce the feed per turn. Similarly, it can be seen that the chip thickness also decreases, so the cutting resistance of the material decreases, and the axial drilling force decreases accordingly.

\section{REFERENCES}

[1] Wang, M.H., Sun, G.Q. (2013), "Finite Element Analysis of the Effect of Chisel Edge on Drilling Composite Laminates", Machinery Design \& Manufacture, Vol.6, pp.237- 243.

\section{BIOGRAPHIE}

Mian Gong, 1963-, has more than 20 years teaching experience in automobile, parts designing and PDM project management. Main research fields: chassis designing, application of $\mathrm{CAD} / \mathrm{CAE}$ in automobile designing and automobile failure diagnosis technology. 